\title{
Movements of Free-Range Pigs in Rural Communities in Zambia: An Explorative Study Towards Future Ring Interventions for the Control of Taenia Solium
}

Inge Van Damme ( $\square$ inge.vandamme@ugent.be)

Ghent University: Universiteit Gent https://orcid.org/0000-0003-2488-1132

lan Pray

Oregon Health \& Science University

Kabemba E. Mwape

University of Zambia School of Veterinary Medicine

Chiara Trevisan

ITG: Instituut voor Tropische Geneeskunde

Fien Coudenys

Ghent University: Universiteit Gent

Chishimba Mubanga

University of Zambia School of Veterinary Medicine

Chembesofu Mwelwa

University of Zambia School of Veterinary Medicine

Victor Vaernewyck

Ghent University: Universiteit Gent

Pierre Dorny

ITG: Instituut voor Tropische Geneeskunde

\section{Seth E. O'Neal}

Oregon Health \& Science University

Sarah Gabriël

Ghent University: Universiteit Gent https://orcid.org/0000-0002-2523-233X

\section{Research Article}

Keywords: Taeniosis, Cysticercosis, Control, GPS, Movement, Sus scrofa; Sub-Saharan Africa; Zambia; Ring treatment

Posted Date: February 2nd, 2022 
DOI: https://doi.org/10.21203/rs.3.rs-1305689/v1

License: (c) (1) This work is licensed under a Creative Commons Attribution 4.0 International License. Read Full License 


\section{Abstract}

Background: Taenia solium typically affects resource-poor communities where pigs are allowed to roam freely, and sanitation and hygiene levels are suboptimal. Sustainable, long-term strategies are urgently needed to control the disease. Geographically targeted interventions, i.e., screening or treatment of taeniosis among people living near infected pigs (defined as ring screening and ring treatment, respectively), have been shown to be effective control options in Peru. However, these results might not be directly generalizable to sub-Saharan African settings. Pig movements play a vital role in the transmission and consequently the success of ring interventions against $T$. solium. Therefore, the aim of the present study was to explore roaming patterns of pigs in T. solium endemic communities in Zambia as a first step to evaluate whether ring interventions should be considered in Zambia.

Methods: In total, 48 free-roaming pigs in two rural neighborhoods in Eastern Province of Zambia were tracked using a Global Positioning System (GPS) receiver. Tracking took place in April 2019 (end of the rainy season) and October 2019 (end of the dry season). The number of revisitations and the time spent within rings of different radii $(50 \mathrm{~m}, 100 \mathrm{~m}$, and $250 \mathrm{~m})$ around the coordinates of the pig owners' household were calculated for each pig.

Results: The tracking time of 43 pigs in the final analysis set ranged between 43 and 94 hours. Pigs spent a median of $31 \%$ and $13 \%$ of the tracked time outside the $50 \mathrm{~m}$ and $100 \mathrm{~m}$ radius, respectively, though large variations were observed between pigs. Overall, 25 pigs (58\%) went outside the $250 \mathrm{~m}$ ring at least once, and individual visits up to 16 hours were observed. In the dry season, 17 out of 23 pigs went outside the $250 \mathrm{~m}$ radius compared to only 8 out of 20 pigs in the rainy season $(p=0.014)$.

Conclusions: In our study sites in Zambia, the majority of pigs spent most of their time within 50 or 100 $\mathrm{m}$ of their owners' home, and results are comparable with those of Peruvian pigs. Both radii could therefore be considered reasonable in future ring interventions.

\section{Background}

People infected with the adult tapeworm of Taenia solium can excrete high numbers of infective eggs [1]. When pigs ingest tapeworm eggs through coprophagia or contaminated food/water, the larvae migrate to tissues and organs, where they mature into cysticerci (porcine cysticercosis). People who consume cysticerci in undercooked pork close the cycle by developing taeniosis. The main public health burden of T. solium results from the fact that humans may become accidental intermediate hosts after ingesting eggs. When the larvae migrate to the central nervous system, they may cause neurocysticercosis, which is an important cause of acquired epilepsy in regions where $T$. solium is endemic [2]. Endemic regions are typically resource-poor communities where pigs are allowed to roam freely and sanitation and hygiene levels are suboptimal, conditions that occur in many rural areas of sub-Saharan Africa, Asia and Latin America. 
Many intervention strategies targeting humans and/or pigs have been described to interrupt the transmission of $T$. solium [3]. Although recent results from endemic areas in Peru and Zambia showed that elimination is possible $[4,5]$, achieving this endpoint required multiple rounds of 4 or 6-monthly human Mass Drug Administration (MDA) combined with pig mass treatment/vaccination, and health education. Because the substantial resources required to achieve or maintain elimination may not be available in most endemic regions, efforts should be made to find effective and sustainable interventions to control the disease. Geographically targeted interventions among people living near visibly infected pigs, using taeniosis treatment (defined as ring treatment) or taeniosis treatment after screening (ring screening), have been shown to be effective control options in Peru [6], where strong clustering of porcine cysticercosis around human taeniosis has been demonstrated [7]. However, pig roaming behaviors may be different in other endemic regions of the world due to differences in housing density, geography, climate, agricultural practices, pig breeds, or open defecation practices, and may consequently require differently sized rings.

Selecting an ideal radius for ring interventions is a balancing act. While larger rings are more likely to capture the source case of taeniosis, they also lose efficiency as the targeted population increases and the intervention approaches the scale of MDA. Smaller radius rings, on the other hand, may risk not capturing the source case of taeniosis. For the region in northern Peru, where the ring interventions were developed, $100 \mathrm{~m}$ rings were chosen as a convenient size for easy field implementation and these were effective when compared to mass applied interventions [6]. If large differences in roaming patterns of pigs are present in Zambia compared to Peru, particularly time spent and frequency of visits outside of feasible ring radii, other control options might be more practical and effective. Knowledge of the pig roaming behavior could also be used to tailor ring interventions to this area. Therefore, the aim of the present study was to explore the roaming patterns of pigs in T. solium endemic communities in Zambia as a first step to evaluate whether ring interventions should be considered in Zambia.

\section{Material \& Methods}

\subsection{Study area and population}

This study took place in the Sinda and Katete Districts of the Eastern Province of Zambia (Additional file 1: Figure S1), where T. solium is endemic [8]. Two rural neighborhoods, participating in a larger T. solium control trial [4], were selected for this study because of the presence of free-roaming pigs and human open defecation in the villages, village accessibility throughout the year, and community willingness to participate. Pigs were tracked in April 2019, which is at the end of the rainy season (presented in this manuscript as 'rainy season'), and October 2019, which is at the end of the dry season (presented as 'dry season'). At each time point, we approached all pig-owning households and requested consenting farmers to provide a pig from their household and include it in the study. Pigs that were 2 months of age or older, healthy, not in late gestation, and that were allowed to roam freely (i.e., not routinely corralled or tethered) were eligible for inclusion. To obtain a representative sample, we aimed to include one eligible pig per pig-owning household. Pigs were selected based on convenience (presence around the household 
at the time of recruitment and ability to catch the pig) and size (according to the available sizes of harnesses). A map of both neighborhoods indicating the households of the participating pigs is shown in Additional file 1: Figure S2.

\subsection{Pig tracking}

Each pig received an ear tag with a unique identifier code, and was fitted an adjustable nylon harness with an "iGotU" portable Global Positioning System (GPS) receiver (MobileAction Technology, New Taipei City, Taiwan) enclosed in a waterproof case (HPRC 1100, Plaber, Vicenza, Italy) [9] (Additional file 1: Figure S3). The time the GPS device was turned on was recorded, along with the household code, and the age and sex of the pig. Signal transmission took place once every minute and pigs were continuously tracked for up to four days. Pigs were monitored daily and any interventions (e.g., harness adjustments) were recorded. On the agreed final day, the starting time of the pursuit and the time the GPS device was turned off were recorded. GPS data of each pig were downloaded using @Trip and were exported to csv files.

\subsection{Data analysis}

All pre-processing steps, visualizations and analyses of the data were done using $\mathrm{R}$ version 4.1.2. [10]. Different pre-processing steps were conducted, balancing between removing inaccurate data and overzealous rejection of valid animal movements [11]. The data from each pig were explored by plotting observations and tracks on maps using the R package ggmap [12], and evident location errors (e.g. points outside the study area) were removed. Points with an unrealistic movement speed were identified using the R package atlastools [13], excluding points with an incoming or outgoing speed above $3 \mathrm{~m} / \mathrm{s}$ [14]. Since data shortly after release and before capture may not properly represent the pig's movement, the first 60 minutes and last 30 minutes were excluded. Additional temporal filters were used whenever an intervention was done (e.g., harness adjustments), by removing 30 minutes before and after the intervention.

\subsubsection{Calculation of revisitation metrics}

The coordinates were projected from latitude-longitude to Universal Transverse Marcator zone 36 south using spTransform from the rgda/ package [15]. Revisitation metrics were calculated using the recurse package [16]. The residence time was determined for every visit outside rings of different radii (50m, $100 \mathrm{~m}, 150 \mathrm{~m}, 200 \mathrm{~m}$, and $250 \mathrm{~m}$ ) around the coordinates of the pig-owning household (which are denoted as ' $50 \mathrm{~m}$ ring' up to ' $250 \mathrm{~m}$ ring'). Household coordinates were determined at the front door of the pig owner's main building, collected during a baseline survey in 2015 [4] using a handheld GPS receiver (eTrex LegendH Cx, Garmin). The proportion of time outside the ring was calculated by dividing the total time outside the ring by the total tracking time. The furthest straight-line distance from the household coordinate was also calculated. 


\subsubsection{Exploring the association of outcomes with predictor variables}

The proportion of time spent outside the rings was analyzed for the $50 \mathrm{~m}$ ring and $100 \mathrm{~m}$ ring using linear regressions. Since a considerable number of pigs did not go outside the $250 \mathrm{~m}$ ring, going outside the $250 \mathrm{~m}$ ring (yes/no) was analyzed as a binary variable, using a logistic regression. The neighborhood, tracking season, and pig sex and age (categorized as $\leq 6$ months or $>6$ months) were included as predictor variables. Different multivariate models were created and were compared using Akaike information criterion (AIC), retaining the model with the lowest AIC. Neighborhood and season were always included in the models to correct for the study design. Assumptions of the linear models were checked using residual plots. For the proportion of time outside the $100 \mathrm{~m}$ ring and the furthest distance travelled, the outcome was log transformed prior to analysis, and transformed back to the original scale for reporting. For all models, marginal effects (predicted values) for the outcome variable were estimated using the function ggeffect [17]. Given the exploratory (hypothesis generating) nature of this study and the relatively low sample size, a significance level of $10 \%$ was used for all tests.

\section{Results}

\subsection{Pig characteristics}

In total, 48 pigs, all of local breed, were enrolled in the study (12 per neighborhood per season). Results for five pigs were excluded due to confinement during the entire tracking time $(n=3$; rainy season in neighborhood A) or due to loss of the GPS device ( $n=2$; one in the dry season in neighborhood $A$, and one in the rainy season in neighborhood $B$ ). The data of 20 pigs tracked in the rainy season and 23 pigs tracked in the dry season were used for the final analyses (Table 1; Additional File 2). Most pigs were female $(35 / 43,81 \%)$ and the age varied between 3 and 71 months, with a median age of 10 months. The pigs in the final analysis set originated from 19 different households in the rainy season and 22 households in the dry season. Two pigs originated from the same household in the rainy season, and two pigs were from the same household during dry season. From nine households, a pig was included in both seasons (four in Neighborhood A, five in neighborhood B). The tracking time of the included pigs ranged between 44 and 95 hours (Table 1 ). 
Table 1

Characteristics and tracking information of pigs included in the final analysis $(n=43)$.

\section{Rainy season}

Neighborhood

$A(n=9)$
Dry season

Neighborhood

$B(n=11)$
Neighborhood

$A(n=11)$
Neighborhood

$B(n=12)$

\section{Sex}

\begin{tabular}{lllll} 
- Female & 6 & 10 & 10 & 9 \\
- Male & 3 & 1 & 1 & 3 \\
\hline
\end{tabular}

Age

- 3-6 months

- 7-11 months

- 12-24 months

- > 24 months

Tracking information

- Median tracking time, in

hours (minimum -

maximum) ${ }^{a}$

- Median number of observations*

- Total number of observations used for analysis

12

6

2

0

2

3

3

3

74 (64-78)

$70(44-91)$

$72(65-77)$

$93(68-95)$

3

4

0

3

5

1

3 a based on entries that were used in the final analysis set (after cleaning)

\subsection{Pig tracking results}

As an illustration, tracking results from four different pigs are visualized in Figure 1. A summary of the proportion of time spent outside different rings is shown in Figure 2. More detailed summary figures are provided in Additional file 1: Figure S4.

None of the pigs stayed within the $50 \mathrm{~m}$ ring around their household throughout the entire tracking period. Overall, pigs spent a median of $31 \%$ of the tracked time outside the $50 \mathrm{~m}$ radius, though large variations were observed between pigs (IQR $=35$, range between $<1 \%$ and $93 \%$; Figure 2 ). The longest visit outside the $50 \mathrm{~m}$ ring ranged between pigs from 13 minutes to 18 hours, with a median of 5 hours. No significant differences were found in the time outside the $50 \mathrm{~m}$ ring between neighborhoods, seasons, sexes and ages $(p>0.10)$. 
Most pigs went outside the $100 \mathrm{~m}$ ring, as only two pigs (5\%) stayed within the $100 \mathrm{~m}$ radius during the entire tracking period. The median time spent outside the $100 \mathrm{~m}$ radius was $13 \%$, ranging from $<1-92 \%$. Six pigs spent more than half of their time outside their $100 \mathrm{~m}$ ring. Older pigs spent more time outside the $100 \mathrm{~m}$ ring $(14 \%[95 \% \mathrm{Cl} 8-23 \%])$ compared to younger pigs $(5 \%[2-11 \%] ; p=0.044)$. No significant differences were found between neighborhoods, seasons, and pig sexes $(p>0.10)$. The median duration of a visit outside the $100 \mathrm{~m}$ ring varied between pigs from 1 minute to 247 minutes (median 10 minutes), and the longest individual visits lasted for 1232 minutes ( $>20$ hours). The visits outside the $100 \mathrm{~m}$ ring showed a bimodal distribution throughout the day, particularly in the dry season, with most visits occurring around 5 a.m. and 4 p.m. (Additional file 1: Figure S5).

Overall, 25 pigs (58\%) went at least once outside the $250 \mathrm{~m}$ ring. Although half of these pigs spent only $2 \%$ or less of their time outside this ring, one pig spent $47 \%$ of its time outside this ring. The median duration of a visit outside the $250 \mathrm{~m}$ ring ranged between pigs from 2 minutes to 214 minutes (median 9 minutes), and individual visits up to 966 minutes (16 hours) were observed.

In the dry season, 17 out of 23 pigs went outside the $250 \mathrm{~m}$ radius compared to only 8 out of 20 pigs in the rainy season $(p=0.014)$. The probability of travelling outside the $250 \mathrm{~m}$ ring in neighborhood $A$ was $82 \%$ [95\% Cl 49-95\%] in the dry season compared to $22 \%$ [6-58\%] in the rainy season. In neighborhood B, the estimated probabilities were $67 \%[38-87 \%]$ in the dry season and $55 \%[27-80 \%]$ in the rainy season.

On average, the maximum straight-line distance away from the household during the tracking period was $280 \mathrm{~m}$ (median value; minimum $100 \mathrm{~m}$, maximum $855 \mathrm{~m}$ ). Two pigs travelled more than $800 \mathrm{~m}$ away from their owners' household. The maximum distance traveled was associated with the age of the pig $(\mathrm{p}=$ 0.075), while correcting for season and neighborhood. The predicted maximum distance was $296 \mathrm{~m}$ [95\% $\mathrm{Cl} 247-357 \mathrm{~m}]$ for older pigs, compared to $214 \mathrm{~m}$ [158-290m] for younger pigs.

\section{Discussion}

In this study, we tracked pigs to explore their movements within and outside rings of different radii around their owners' household, as a step towards developing ring treatment strategies for the control of $T$. solium in rural communities in Zambia. We found that the majority of pigs spent most of their time inside both a $50-\mathrm{m}$ radius and $100 \mathrm{~m}$ radius around their owner's home, with a median of $69 \%$ and $87 \%$ of the time, respectively. This is very similar to the study of Pray and colleagues [9], which found that pigs in Peru spent a median of $70 \%$ and $83 \%$ of their roaming time within $50 \mathrm{~m}$ and $100 \mathrm{~m}$, respectively, of their homes. Since 100m ring interventions were shown to be efficient in Peru [6], the similarity in residence time between our findings and those in Peru [9] indicates that a $100 \mathrm{~m}$ ring intervention may also be effective in Sub-Saharan rural settings.

The majority of pigs (95\%) in Zambia roamed outside the $100 \mathrm{~m}$ ring around their homes at least once, and more than half of the pigs travelled more than $250 \mathrm{~m}$ away from their home, providing ample opportunity to become infected beyond the $100 \mathrm{~m}$ ring. If a pig were infected greater than $100 \mathrm{~m}$ from their home, the source case of taeniosis would not be identified or treated. Consequently, this would advocate 
for increasing the ring radius to reach the desired control effect. Nevertheless, this study was limited to observing pig roaming patterns, which may not be indicative for the exposure with $T$. solium eggs and thus the risk of infection. Many other factors could influence pig risk of infection and the potential effectiveness of ring interventions.

Factors that influence pig movement can vary between regions and even between villages in the same region. We found that pigs were more likely to roam outside a $250 \mathrm{~m}$ ring in the dry season compared to pigs in the rainy season. This could be related to the lower availability of food in the dry season, and therefore the need to roam further from home. Similar patterns were observed in rural Kenya, where larger (non-significant) home ranges were observed in the dry season compared to the rainy season [18]. In contrast, in Peru, almost all occurrences of extended roaming were observed in the rainy season, and home ranges of Peruvian pigs were $61 \%$ larger during the rainy season than in the dry season [14]. We also have some indications that older or male pigs travel longer distances and more frequently roam outside the $100 \mathrm{~m}$ ring. In Peru, long-distance roaming pigs had more contact with open defecation areas and are thus an important subgroup in the context of interventions to control T. solium [14]. Differences in pig roaming behavior were also observed between villages in the same region in Peru [14]. Likewise, in the present study, there were some indications that pigs in neighborhood $A$ traveled less frequently outside the $250 \mathrm{~m}$ ring compared to pigs in neighborhood $\mathrm{B}$, which could potentially be explained due to the closer proximity of the households within the community in neighborhood $A$. In the latter neighborhood, one round of ring strategy with a few $100 \mathrm{~m}$ rings would approximate an MDA. Therefore, defining the optimal ring size is a balance between being sufficiently large to obtain the desired effectiveness, while also being the smallest possible to increase efficiency. Assuming that there is a relation between residence time and the risk of infection, the $50 \mathrm{~m}$ ring could potentially be a worthy alternative.

This was an exploratory study with limitations. Although active foraging time may be a better proxy for the infection risk with $T$. solium, we didn't exclude night or inactive time points, as several pigs in our study roamed during the night, and GPS data are not reliable to assess activity [19]. The use of collarmounted activity sensors or visual observations may be useful to provide more insights in animal activity and behavior, including the assessment of pig exposure to human stool and the $T$. solium infection risk. The pigs in our sample could be weaker and smaller compared to the total population as they were selected based on their size (according to the available harnesses) and convenience (opportunity to catch the pigs), potentially resulting in a sample of weaker, smaller pigs, and those who were at home at time of enrolment (i.e., less likely to be roamers). Given the relatively short tracking time of certain pigs ( $<48 \mathrm{~h})$, we also cannot completely rule out the possibility that capture, harness or unforeseen events may have affected the pigs' normal behavior. Although the relatively small sample size and inclusion of only two villages limits the strength of conclusions that can be made, this study is important to build the necessary knowledge base for trialing and tailoring ring interventions to this new setting.

\section{Conclusions}


In our study sites in Zambia, the majority of pigs spent most of their time within 50 or $100 \mathrm{~m}$ of their owners' home. Our data support the previously proposed $100 \mathrm{~m}$ ring interventions, although a $50 \mathrm{~m}$ radius may also be considered reasonable in future interventional trials. While studies to evaluate the strength and extent of clustering of human taeniosis and porcine cysticercosis could provide additional information [7], this requires complete screening of entire villages for taeniosis, as well as purchase and necropsy of all pigs; both expensive endeavors. Resources might better be applied towards completion of an intervention trial, as this is ultimately needed to determine whether ring interventions can achieve the right combination of effectiveness and efficacy in Zambia.

\section{Abbreviations}

AIC

Akaike information criterion

GPS

Global Positioning System

IQR

inter-quartile range

MDA

Mass Drug Administration.

\section{Declarations}

\subsection{Acknowledgements}

We acknowledge all pig owners for their participation in the study.

\subsection{Funding}

The study received financial support from the Institute of Tropical Medicine, Antwerp (https://www.itg.be) via the Flemish Department of Science, Economy and Innovation (EWI, https://www.ewi-vlaanderen.be/) and via the Belgian Development Cooperation (DGD, https://diplomatie.belgium.be/en/dgd) in the framework of the collaboration between the Institute of Tropical Medicine in Antwerp, Belgium, and the University of Pretoria, South Africa. The funders had no role in study design, data collection and analysis, decision to publish, or preparation of the manuscript.

\subsection{Availability of data and materials}

The individual data points, tracks and summary statistics per pig are provided in Additional file 2: GPS tracks and summary data of individual pigs. Exact location data used for analyses in this manuscript can be accessed upon request via the Institute of Tropical Medicine data access committee. 


\subsection{Authors' contributions}

SEO and SG conceptualized the study. IP, KEM, CT, FC, VV, PD, SEO and SG planned and carried out the study. IVD and IP performed the analyses and visualizations. All authors discussed the results and contributed to the final manuscript.

\subsection{Ethics approval and consent to participate}

The CYSTISTOP project was approved by institutional review boards and ethics committees at the University of Zambia (Lusaka, Zambia) and the University of Antwerp and the Institute of Tropical Medicine (both in Antwerp, Belgium), including the amendment of the pig tracking component (Ref. No. 004-09-15). Oral and written informed consent was obtained from all pig owners participating in the present study.

\subsection{Consent for publication}

Not applicable.

\subsection{Competing interests}

The authors declare that they have no competing interests.

\section{References}

1. García HH, Gonzalez AE, Evans CAW, Gilman RH. Taenia solium cysticercosis. Lancet. 2003;362:547-56.

2. Mwape KE, Blocher J, Wiefek J, Schmidt K, Dorny P, Praet N, et al. Prevalence of neurocysticercosis in people with epilepsy in the Eastern Province of Zambia. PLoS Negl Trop Dis. 2015;9:e0003972.

3. de Coster T, Van Damme I, Baauw J, Gabriël S. Recent advancements in the control of Taenia solium: A systematic review. Food Waterborne Parasitol. 2018;13:e00030.

4. Gabriël S, Mwape KE, Hobbs EC, Devleesschauwer B, Van Damme I, Zulu G, et al. Potential elimination of active Taenia solium transmission in Africa. N Engl J Med. 2020;383:396-7.

5. Garcia HH, Gonzalez AE, Tsang VCW, O'Neal SE, Llanos-Zavalaga F, Gonzalvez G, et al. Elimination of Taenia solium transmission in Northern Peru. N Engl J Med. 2016;374:2335-44.

6. O’Neal SE, Pray IW, Vilchez P, Gamboa R, Muro C, Moyano LM, et al. Geographically targeted Interventions versus mass drug administration to control Taenia solium cysticercosis, Peru. Emerg Infect Dis. 2021;27:2389-98. 
7. Pray IW, Ayvar V, Gamboa R, Muro C, Moyano LM, Benavides V, et al Spatial relationship between Taenia solium tapeworm carriers and necropsy cyst burden in pigs. Brindley PJ, editor. PLoS Negl Trop Dis [Internet]. 2017;11:e0005536. Available from: https://dx.plos.org/10.1371/journal.pntd.0005536.

8. Gabriël S, Mwape KE, Phiri IK, Devleesschauwer B, Dorny P. Taenia solium control in Zambia: The potholed road to success. Parasite Epidemiol Control. 2019;4:e00082.

9. Pray IW, Swanson DJ, Ayvar V, Muro C, Moyano LM, Gonzalez AE, et al. GPS tracking of free-ranging pigs to evaluate ring strategies for the control of cysticercosis/taeniasis in Peru. PLoS Negl Trop Dis. 2016;10:1-14.

10. R Core Team. R: A language and environment for statistical computing [Internet]. Vienna, Austria: $R$ Foundation for Statistical Computing; 2021. Available from: https://www.r-project.org/.

11. Gupte PR, Beardsworth CE, Spiegel O, Lourie E, Toledo S, Nathan R, et al. A guide to pre-processing high-throughput animal tracking data. J Anim Ecol [Internet]. 2021;00:1-21. Available from: https://onlinelibrary.wiley.com/doi/10.1111/1365-2656.13610.

12. Kahle D, Wickham H. ggmap: Spatial visualization with ggplot2. R J. 2013;5:144-61.

13. Gupte P. atlastools: Tools for pre-processing high-throughput animal tracking data. 2021.

14. Pray IW, Muro C, Gamboa R, Vilchez P, Wakeland W, Pan W, et al. Seasonal patterns in risk factors for Taenia solium transmission: A GPS tracking study of pigs and open human defecation in northern Peru. Parasites Vectors. 2019;12:1-12.

15. Bivand R, Keitt T, Rowlingson B. rgdal: Bindings for the "Geospatial" data abstraction library [Internet]. 2019. Available from: https://cran.r-project.org/package=rgdal.

16. Bracis C, Bildstein KL, Mueller T. Revisitation analysis uncovers spatio-temporal patterns in animal movement data. Ecography. 2018;41:1801-11.

17. Lüdecke D. ggeffects: Tidy data frames of marginal effects from regression models. J Open Source Softw. 2018;3:772.

18. Thomas LF, De Glanville WA, Cook EA, Fèvre EM. The spatial ecology of free-ranging domestic pigs (Sus scrofa) in western Kenya. BMC Vet Res. 2013;9:1-12.

19. Petroelje TR, Belant JL, Beyer DE, Svoboda NJ. Identification of carnivore kill sites is improved by verified accelerometer data. Anim Biotelemetry. 2020;8:18.

\section{Figures}




\section{Image not available with this version}

\section{Figure 1}

*The preprint version of figure 1 was removed due to potential identifiers. *

Visualization of pig tracks.

The circles represent different rings around the pig-owning household (green central point): $50 \mathrm{~m}$ ring (inner circle), $100-\mathrm{m}$ ring (middle circle), and $250 \mathrm{~m}$ ring (outer circle). Pig a) spent $88 \%, 55 \%$ and $0 \%$ of its time outside the $50-\mathrm{m}, 100-\mathrm{m}$, and $250-\mathrm{m}$ ring, respectively; b) spent $19 \%, 15 \%$ and $1 \%$ of its time outside the $50-\mathrm{m}, 100-\mathrm{m}$, and $250-\mathrm{m}$ ring, respectively; c) spent $22 \%, 16 \%$ and $11 \%$ of its time outside the $50 \mathrm{~m}$, $100 \mathrm{~m}$, and $250-\mathrm{m}$ ring, respectively; d) spent $73 \%, 68 \%$ and $1 \%$ of its time outside the $50 \mathrm{~m}, 100 \mathrm{~m}$, and $250 \mathrm{~m}$ ring, respectively. Each color represents a different day. 


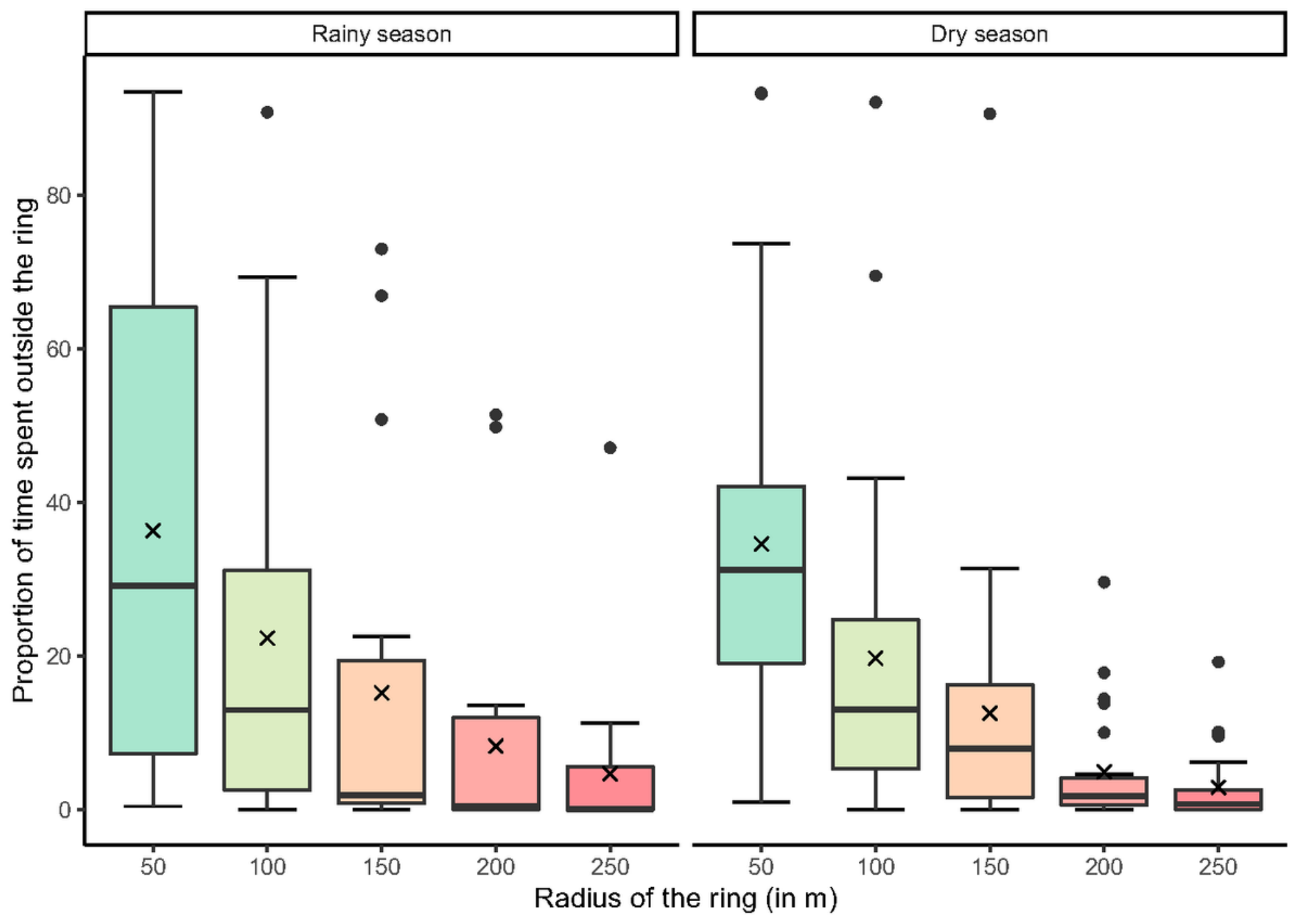

Figure 2

Proportion of the tracking time pigs spent outside different ring sizes around their household $(n=43$ pigs).

The cross-mark $(x)$ represents the mean value, the horizontal line in the box the median value, the lower and upper hinges the first and third quartiles. The whiskers extend to the largest value no further than 1.5 times the inter-quartile range (IQR) and the individual dots represent observations above the latter value.

\section{Supplementary Files}

This is a list of supplementary files associated with this preprint. Click to download.

- Additionalfile2.pdf

- paperGPSadditionalfile120220323.pdf

- graphicalabstract20220323.pdf 